\title{
An Overwhelming Desire to Be Blind: Similarities and Differences between Body Integrity Identity Disorder and the Wish for Blindness
}

\author{
Katja Gutschke ${ }^{a}$ Aglaja Stirn ${ }^{b}$ Erich Kasten $^{c}$ \\ ${ }^{a}$ Medical School Hamburg, University of Applied Sciences, Hamburg, Germany; \\ ${ }^{b}$ Klinik für Psychosomatik und Psychotherapie, Universitätsklinikum Schleswig-Holstein, \\ Kiel, Germany; 'Department of Neuropsychology, Medical School Hamburg, \\ Hamburg, Germany
}

\section{Keywords}

Blindness · Body integrity identity disorder

\begin{abstract}
Background: The urge to be permanently blind is an extremely rare mental health disturbance. The underlying cause of this desire has not been determined yet, and it is uncertain whether the wish for blindness is a condition that can be included in the context of body integrity identity disorder, a condition where people feel an overwhelming need to be disabled, in many cases by amputation of a limb or through paralysis. Objective: The aim of this study is to test the hypothesis that people with a desire for blindness suffer from a greater degree of visual stress in daily activities than people in a healthy visual control group. Method: We created a Likert scale questionnaire to measure visual stress, covering a wide range of everyday situations. The wish for blindness is extremely rare and worldwide only 5 people with an urge to be blind were found to participate in the study ( 4 female, 1 male). In addition, a control group of 35 ( 28 female, 7 male) visually healthy people was investigated. Questions
\end{abstract}


addressing issues that may be experienced by participants with a desire to be blind were integrated into the questionnaire. Results: The hypothesis that people with a desire for blindness suffer from a significantly higher visual overload in activities of daily living than visually healthy subjects was confirmed; the significance of visual stress between these groups was $p<0.01$. In addition, an interview with the 5 affected participants supported the causal role of visual overload. Conclusions: The desire for blindness seems to originate from visual overload caused by either ophthalmologic or organic brain disturbances. In addition, psychological reasons such as certain personal character traits may play an active role in developing, maintaining, and reinforcing one's desire to be blind.

(C) 2017 The Author(s)

Published by S. Karger AG, Basel

\section{Introduction}

Body Integrity Identity Disorder and the Desire to Be Blind

Body integrity identity disorder (BIID) is a rare mental disorder characterized by an intensive desire to adapt the body to a mentally perceived body image. Affected people often feel that an arm or a leg does not belong to their body and ask physicians for an amputation [1-10]. Currently, conditions such as the desire for paraplegia or - more rarely - for blindness or deafness are being discussed in the context of BIID. The incidence of BIID is estimated to be several thousand people worldwide [11,12], a figure partly based on the number of affected participants on relevant Internet forums. Fewer people are affected by the desire to be blind; official estimations of its frequency are not currently available.

The exclusion criteria for BIID include psychotic disorders such as schizophrenia or delusional disorder [4]. Most affected people participating in studies like this are well educated and occupy prestigious jobs [10] and they actively hide this desire from their social environment. The possible fulfillment of their desire raises a number of serious moral and ethical issues [12, 13]. A study by Noll and Kasten [14] showed that sufferers who fulfilled their need for amputation had no regrets. Based on current studies and research, psychotherapy does not change the sufferers' underlying need but improves their coping strategies with these desires [15].

A 30-year-old American woman, who openly confessed her wish for blindness, recently brought the phenomenon of harboring a desire for blindness to the attention of the general public. In the same time frame, an affected 38-year-old woman gave an interview on Swiss television regarding this phenomenon. It is unknown whether the desire for blindness is generally connected with BIID $[6,9,10]$; particularly, the interaction of neurological, psychological, and sexual components in BIID is not automatically transferable to the desire for blindness.

However, one aspect that is transferable is that affected people are uncomfortable with their sensory perception in the same fashion that people affected by BIID are with a part of their body. In 2006, Veale [16] described a woman who had a compelling desire for deafness, and speculated this desire to be "a severe form of avoidance behavior from hyperacusis and misophonia." Hypersensitivity could result from illness, stress, and several external circumstances. The wish to be blind is such a serious wish that one might assume that it is not 


\section{Case Reports in Ophthalmology}

(C) 2017 The Author(s). Published by S. Karger AG, Basel www.karger.com/cop

Gutschke et al:: An Overwhelming Desire to Be Blind: Similarities and Differences between Body Integrity Identity Disorder and the Wish for Blindness

caused by a partial overload (e.g., reading small text). A more supportable assumption would be that the wish to be blind is caused by a steady visual overload and that the affected people might feel relieved in the state of blindness. The permanent impairment of everyday visual processes could very well be a contributing factor to the wish for blindness. We describe this factor with the term "visual overload."

\section{Visual Stress}

There is no common terminology for the concept of "visual overload." In the literature, more or less interchangeable terms such as "visual stress," "visual fatigue," and "visual discomfort" can be found. The last of these is listed in the US-specific version of the ICD (H53.14) as a subcategory of visual disturbances [11] and is often associated with photosensitivity and problems with reading and writing. Conlon et al. [17] developed a tool to measure "visual discomfort" and reported that about $12 \%$ of their group had a high degree of visual discomfort. Based on the same test, Borsting et al. [18] found a prevalence of about $1 \%$ in people who suffer from visual stress. Women are more likely to experience high visual stress than men [18].

Based on our assumption that a permanent feeling of visual overload in everyday life causes the desire for blindness, we have developed a questionnaire that focuses on this theory. Our first step was to search for symptoms of visual overload in several aspects of everyday life and incorporate them into the questionnaire.

Situations such as a rapid change of strong visual stimuli, flickering lights or extended exposure to TV or computer screens can cause physical symptoms in people with increased sensitivity [19]. Damage to the visual system in the brain (acquired at birth or later in life) can often lead to visual fatigability [20], impairment of contrast sensitivity [21], impairment of light/dark adaptation [20], double vision [22], and heterophoria [22]. Congenital weakness is classified as minimal cerebral dysfunction, which also includes the Meares-Irlen syndrome. The symptoms of this syndrome [23] include general hypersensitivity to light, disturbed perception of space with misjudgment of distances and object relationships, and excessive strain and visual exertion, which alters the appearance of letters and words (that is, they appear to be moving, blurred and deformed). The tendency towards headaches, migraines, eye pain, and problems with reading are some of the symptoms. The Meares-Irlen syndrome is associated with dyslexia [21].

\section{Methods}

To test our assumption, we formulated two hypotheses: (1) people who wish to be blind are more likely to suffer from visual stress than visually healthy people; (2) the intensity of the desire to be blind correlates with the intensity of subjectively experienced visual overload.

\section{Development of the Visual Stress Inventory}

To measure visual stress, we developed a questionnaire containing 5 everyday categories. These include the symptoms of Meares-Irlen syndrome, minimal cerebral dysfunctions, 


\section{Case Reports in Ophthalmology}

and general visual stress. The categories are as follows: (1) general questions about visual stress, (2) daily routine, (3) reading and writing, (4) road traffic, and (5) motion/movement.

The Visual Stress Inventory (VSI) contained 75 items. Cronbach's $\alpha$ of the subtest questions was sufficient: general visual stress, 0.83 ; daily routine, 0.87 ; reading and writing, 0.93; road traffic, 0.88; motion/movement, 0.92; all, 0.96. In addition, we developed another experimental test in the form of a hidden object game to assess visual processing speed.

There are inventories assessing overload of sensory stimuli [24], but they were not appropriate and detailed enough to get a comprehensive result concerning visual discomfort. Moreover, we did not use a neuropsychological object recognition test (e.g., Hooper's Visual Organization Test [25] or Kaufman's gestalt closure [26]), because neuropsychological tests for visual agnosia were considered as not sufficiently sensitive to our research question and furthermore, affected people have no problems with object recognition.

\section{Sample}

The survey utilized the online tool "SoSciSurvey" and was available in both German and English. A link to the survey was posted on several Internet forums to increase participation within the unaffected control group. Five people with the desire to be blind ( 4 female, 1 male) and 41 control subjects ( 28 female, 13 male) participated in the investigation. In order to take the influence of gender into account, the control group was modified by random deletion of male participants to achieve the same gender ratio as in the affected group (1 male: 4 female; control group: 7 male:28 female). Participants of the control group were $41.4 \pm 19.1$ years old, while participants of the affected group were $38.2 \pm 12.5$ years old.

\section{Test of Hypothesis I}

Using the Mann-Whitney test, we examined whether the particular stress values in the 5 categories and the total stress value of affected people were different from those in the control group. The hidden object test results were then analyzed separately.

\section{Test of Hypothesis II}

Additional questions were created to address the condition of those with a desire for blindness. For an investigation of hypothesis II, the correlation between the intensity of the desire for blindness and the degree of visual overload was calculated. Because of the comparability to BIID, a validated and standardized questionnaire for BIID [27] was used to determine the intensity of their desire for blindness. We modified the original test by Fischer et al. [27] by using terms related to blindness.

\section{Additional Questions Concerning Qualitative Evaluation}

In addition, we asked questions to collect information about eye diseases and their treatment, as well as about reinforcing situations that may have affected one's desire for blindness. Furthermore, we asked participants to report the age at which they first felt the desire to be blind and to report whether they had planned or carried out self-inflicted behavior to obtain blindness. This also allowed a comparison between those who wish to be blind and BIID sufferers. 


\section{Case Reports in Ophthalmology}

\section{Results}

\section{Hypothesis I}

The first hypothesis postulates that those who wish to be blind experience a higher degree of visual stress than participants in the control group. We investigated the intensity of visual stress in the 5 categories described above: (1) general questions on visual stress, (2) daily routine, (3) reading and writing, (4) road traffic, and (5) motion/movement. All questions were set on an 11-point Likert scale (from $1=$ no visual stress to $11=$ maximum visual stress). We were then able to calculate a total stress score based on all categories (Table 1; Fig. 1, Fig. 2).

The Mann-Whitney test showed that those affected with a desire for blindness experienced a significantly higher stress level compared to those in the control group. Thus, hypothesis I was confirmed $(p<0.01)$. In 3 of the 5 categories (everyday routine, reading and writing, and movement), the score of those affected with a desire to be blind was significantly higher. No significant difference was found in the category "road traffic." The results also showed an impact of gender on visual stress. Male participants scored lower in all subcategories - in 2 subcategories even significantly lower - than female participants.

\section{Hidden Object Game}

The time period within which participants found the objects in 4 hidden object game trials was $152.3 \pm 78.6 \mathrm{~s}$ for those with a desire for blindness $(n=5)$ (Fig. 3). This was significantly higher $(p<0.05)$ than in the control group (87.2 $\pm 36.8 \mathrm{~s} ; n=28)$.

\section{Hypothesis II}

As mentioned above, we utilized a modified version of the Fischer test to measure the intensity of the desire for blindness [27]. Table 2 shows the results.

The intensity of the desire for blindness correlated in 4 out of 5 participants $(A, B, C, E)$ with the intensity of visual stress. However, the participant with the strongest desire for blindness was the one with the lowest visual stress value. Due to this outlier, there was on average only a very small correlation between the intensity of one's desire for blindness and the intensity of visual stress. The value of Kendall's $\tau$ is 0.2 ; thus, the correlation is not significant. Hypothesis II was not confirmed.

\section{Additional Questions}

Four of the 5 participants of the first group (wish for blindness) reported disturbances in their visual system; e.g., "my last prescription was stronger than it had to be, and the stress could be from that" (participant A), "strong wide-sightedness (far-sightedness) with +5 diopters in both eyes" and "sicca syndrome" (participant B), "I got eyeglasses at 12 because of UV damage that caused myopia" (participant D), and "myopia with -5 diopters, astigmatism, squinting since birth, and operation due to the latter" (participant E).

In all 5 participants, the wish to be blind first occurred in childhood, at an average age of about 9 years $(A=8$ years, $B=$ more than 30 years ago [current age: 47 years]; $C=8-10$ years, $\mathrm{D}=9$ years, $\mathrm{E}=$ about 4 years). None of the participants claimed to have made an attempt to become blind with "hard" methods (by rough external influence, such as cauterization). Two of the participants reported that they have stared at the sun in order to induce 


\section{Case Reports in Ophthalmology}

(C) 2017 The Author(s). Published by S. Karger AG, Basel www.karger.com/cop

Gutschke et al:: An Overwhelming Desire to Be Blind: Similarities and Differences between Body Integrity Identity Disorder and the Wish for Blindness

blindness. Another participant reported that he had developed a plan; however, he had never executed it.

Based on a list, which also contains other potential influences on one's desire for blindness, apart from the visual overload, affected participants assessed the following 3 situations to be the most reinforcing on a 7-point Likert scale (from $1=$ strongly disagree to $7=$ strongly agree): (1) brightness of the environment, (2) amount of visual impressions, and (3) being among many people.

An open question on reinforcing situations revealed the following answers:

Participant A: When I'm stressed; when I'm exposed to bright light or complex patterns; when I see blind people, especially if they are doing something.

Participant B: When I watch movies with blind characters, when I read books about blind people, or when I am with my blind girlfriend.

Participant C: Foreign and unfamiliar surroundings, many people around me, hectic atmosphere (e.g., railway station, airport).

Participant D: Walking down the street; doing anything in the dark; being with a blind person; watching TV.

Participant E: Actually always.

Items in the VSI with Highly Significant Group Differences

Responses to the following questions differed significantly between affected participants and those in the control group ( $p<0.001)$ : (1) I feel overburdened by normal vision; (2) I can badly estimate distances and spaces; (3) I avoid bright sunlight; (4) one cannot do it right for me: I feel dazzled despite just even sufficient light; (5) I have difficulty in keeping to the lines when writing; (6) the above problems frequently occurred in my childhood (items concerning reading and writing); (7) if I have to pick up a lot of visual information at a large station (advertising, people, shops and train timetables), I feel uncomfortable; and (8) I often cannot find the desired products in a supermarket shelf.

\section{Discussion}

The task of this study was to answer the following question: can we attribute the desire for blindness to visual overload? A significant finding in the results of the study was that the visual stress value of affected participants is higher $(p=0.004)$ than that of the control group. In 4 subcategories ("daily routine," "reading and writing," "motion/movement," and "general questions on visual stresses"), it was significantly higher. The exception to this differentiation was "road traffic," where there was no significant difference between the affected and the control group.

Affected participants needed significantly more time than the control group to complete the hidden object test. The significant differentiation that became evident within various everyday categories could be an indication that the symptoms of visual stress are not limited to areas such as light sensitivity or difficulty in reading and writing.

Visual stress can be attributed to conditions such as an ophthalmic disease or an organic brain defect. We postulate that the symptoms caused by such a defect in turn enhance the desire for blindness. The group of affected participants had a much higher rate of occurrence 


\section{Case Reports in Ophthalmology}

Case Rep Ophthalmol 2017;8:124-136

DOI: $10.1159 / 000456709$ (10.1159/000456709

(c) 2017 The Author(s). Published by S. Karger AG, Base www.karger.com/cop

Gutschke et al.: An Overwhelming Desire to Be Blind: Similarities and Differences between Body Integrity Identity Disorder and the Wish for Blindness

of visual disorders. This is parallel to BIID, where affected people who want to have an amputation often had injuries of these limbs at some point in their childhood $[8,10,12]$.

Another parallel is that in both groups (amputation/blindness), the desire first appeared in childhood ("problems of reading and writing frequently occurred in my childhood," wrote one participant). Practically all affected subjects have affirmed this in significant contrast to the control group where the situation was almost unheard of. This is an indication that visual overload had already impacted affected subjects during their childhood. Visual stress has a general negative impact not only on reading and writing but it is also presumably an influencing variable over one's whole lifetime. Therefore, we suggest that this explains the emergence of the desire for blindness.

This obvious relationship between visual stress and the desire for blindness also suggests that visual overload reinforces and maintains one's wish to be blind. For example, one participant wrote: "I feel overburdened by normal vision."

From a list of situations where subjects were asked to rate to what degree they reinforce their desire for blindness, affected subjects assessed the following 3 to be the most reinforcing: (1) brightness of the environment; (2) amount of visual impressions; and (3) being among many people. The presumption that light sensitivity is an important factor is also strengthened by the fact that those affected by the desire for blindness agreed more frequently with the following statements than the control group: "I avoid bright sunlight" and "one cannot do it right for me: I feel dazzled despite just even sufficient light."

Thus, bright light is reinforcing and darkness is mitigating for visual stress [18, 19, 23], especially with regard to the symptoms of the Meares-Irlen syndrome [23]. Two of the affected participants named intensive and visually stressful situations as having an impact on their desire for blindness.

That "being among many people" has been rated as a reinforcing situation suggests that there is a hypersensitivity to social stimuli [28]. However, it has to be taken into account that 3 of the affected participants reported to be triggered by encounters with blind people. This shows that beyond visual stress, psychological motives are relevant; the same is the case for BIID patients with a desire for amputation.

With regard to gender differences, Borsting et al. [18] detected a clearly higher risk of visual stress in women. Gender differences of visual perception are reported in the study of Tokaj [29] as well. Our study supports the result that women are more affected by visual stress than men. One might assume that a difference between BIID and the desire for blindness is that BIID predominantly affects men, especially the wish for an amputation, while the desire for blindness seems to occur more often among females. This is hard to confirm because of the low number of affected people. If visual stress is a substantial factor behind the emergence of the desire for blindness, then the higher risk among females could explain the fact that the affected people are predominantly female.

Just as BIID is probably the result of several combining factors leading to the disease, the same could be possible in the case of the desire for blindness. Four out of the 5 participants affected by a desire to be blind were affected predominantly by visual stress and the 1 outlier was predominantly affected by another factor.

Obviously, there are some limitations of the study. It is questionable if the result gained from this tiny number of affected participants represents the situation for all affected people. The study, however, should provide an effective approach to investigate the role of visual 
overload in the development of the wish to be blind. Unfortunately, due to a lack of financial support and the short timeframe, a comprehensive investigation of sensory overload has been beyond the scope of this study. This should be considered in follow-up studies to gain a broader approach to the topic and to reduce suggestibility.

Concerning the second hypothesis, the small number of participants proved to be quite problematic. Because a single participant showed contrary results, no uniform results were found. This lack of results may hint that visual stress is not the leading cause for the wish to be blind. If studies were to be carried out at a larger scale, this hypothesis would have the opportunity to be proven.

Hypersensitivity is found in all sensory systems, i.e., hearing, smell, taste, and skin sensibility [30]. For example, in the auditory system, this can lead to a phobia for loud noises [31], the "supertaster" has an extreme taste [32], and auditory hypersensitivity is often found in autistic children [33]. Even electromagnetic hypersensitivity was reported [34]. People with such a hypersensitivity avoid the corresponding stimuli. The desire for blindness, however, cannot be explained with such oversensitivity alone, and additional factors must be added.

\section{Conclusion}

In summary, the results of our study show a significant correlation between subjective visual stress and the desire for blindness. This preliminary result supports the hypothesis that visual overload plays a role in developing, maintaining, and reinforcing one's desire for blindness.

The study confirmed that people desiring blindness have quite a bit in common with those who suffer from BIID, particularly in terms of early manifestation of the desire. The intensity of the desire for blindness, measured by a modification of the standardized and validated Fischer scale for BIID [27], was strong in 1 case, medium in 3 cases, and weak in 1 case (Table 2). As in amputation BIID cases, those who desire blindness also reported that they had considered or undertaken behavior aimed at bringing about the desired impairment. Based on these similarities, we hypothesize that similar variables play their part in manifesting the desire for blindness and other physical impairments.

Previous causal investigations concerning BIID have established that a combination of psychological, neurological, and, in some cases, sexual components can lead to a desire for disability [8]. In contrast, the study at hand supports the hypothesis that a tendency towards visual stress is essential for the emergence of a desire for blindness. This suggests that the phenomenon is rather related to the medical or neurological than the psychological field.

The extreme rarity of a desire for blindness, in combination with the relatively high prevalence of visual stress [18], shows that other etiologic and perpetuating factors must be relevant. It is possible that factors that are unique to an individual may initiate the desire for blindness or more generally, the desire for disability, but in every case one or more factors consistently influence and maintain the desire. Additional research is necessary to examine and confirm these factors in a larger group. 


\section{Acknowledgement}

We thank all participants of this study and Mr. George Riley for his help in translating.

\section{Statement of Ethics}

The authors have no ethical conflicts to disclose.

\section{Disclosure Statement}

The authors have no conflicts of interest to declare.

\section{References}

1 Brang D, McGeoch PD, Ramachandran VS: Apotemnophilia: a neurological disorder. Neuroreport 2008;9:1305-1306

2 Brugger P, Lenggenhager B, Giummarra MJ: Xenomelia: a social neuroscience view of altered bodily self-consciousness. Front Psychol 2013;4:204.

3 Bruno RL: Devotees, pretenders and wannabes: two cases of factitious disability disorder. Sex Disabil 1997;15:243-260.

First MB: Desire for amputation of a limb: paraphilia, psychosis, or a new type of identity disorder. Psychol Med 2005;35:919-928.

Giummarra MJ, Bradshaw JL, Hilti LM, Nicholls ME, Brugger P: Paralyzed by desire: a new type of body integrity identity disorder. Cogn Behav Neurol 2012;25:34-41. Johnson AJ, Liew SL, Aziz-Zadeh L: Demographics, learning and imitation, and body schema in body integrity identity disorder. J Cogn Sci 2011;6:8-15.

7 Kasten E: Body integrity identity disorder; in Schnell T (ed): Praxisbuch: Moderne Psychotherapie: Der Guide bei komplexen Störungsbildern. Berlin, Springer, 2016, pp 49-72. Fortschr Neurol Psychiatr 2009;77:16-24. Sedda A, Bottini G: Apotemnophilia, body integrity identity disorder or xenomelia? Psychiatric and neurologic etiologies face each other. Neuropsychiatr Dis Treat 2014;10:1255-1265. Stirn A, Thiel A, Oddo S: Body Integrity Identity Disorder (BIID): Störungsbild, Diagnostik, Therapieansätze. Weinheim, Beltz, 2010.

11 Buck CJ: 2016 ICD-10-CM Standard Edition. Amsterdam, Elsevier Health Sciences, 2015.

12 Müller S: Body Integrity Identity Disorder (BIID) - Ist der Amputationswunsch eine autonome Entscheidung oder Ausdruck einer neuropsychologischen Störung?; in Gross D, et al: Normal - anders krank? Berlin, Medizinisch Wissenschaftliche Verlagsgesellschaft, 2007, pp 229-266. Bayne T, Levy N: Amputees by choice: body integrity identity disorder and the ethics of amputation. J Appl Philos 2005;22:75-86.

14 Noll S, Kasten E: Body integrity identity disorder (BIID): how satisfied are successful wannabes? Psychol Behav Sci 2014;3:222-232.

15 Kröger K, Schnell T, Kasten E: Effects of psychotherapy on patients suffering from body integrity identity disorder (BIID). Am J Appl Psychol 2014;3:110-115.

16 Veale D: A compelling desire for deafness. J Deaf Stud Deaf Educ 2006;11:369-372.

17 Conlon EG, Lovegrove WJ, Chekaluk E, Pattison PE: Measuring visual discomfort. Vis Cogn 1999;6:637663.

18 Borsting E, Chase CH, Ridder WH: Measuring visual discomfort in college students. Optom Vis Sci 2007;84:745-751.

19 Hellbrück J, Fischer M: Umweltpsychologie: Ein Lehrbuch. Göttingen, Hogrefe, 1999. 
Niedeggen M, Jörgens S: Visuelle Wahrnehmungsstörungen. Göttingen, Hogrefe, 2005.

Zihl J, Priglinger S: Sehstörungen bei Kindern: Diagnostik und Frühförderung. Berlin, Springer, 2013.

Kaufmann H, Steffen H: Strabismus. Stuttgart, Thieme, 2012.

Safra D, Steiner F: Irlen-Syndrom, Asthenopie und Leseschwäche. Schweiz Z Heilpädagogik 2005;2:1017.

24 Barsky AJ, Wyshak G, Klerman GL: The somatosensory amplification scale and its relationship to hypochondriasis. J Psychiatr Res 1990;24:323-334. Hooper HE: Visual Organization Test. Los Angeles, Western Psychological Services, 1983. Melchers P, Schürmann S: Gestalterschliessen. Kaufman - Neuropsychologischer Kurztest, dt. Version. Leiden, PITS BV, 2004.

27 Fischer ML, Kasten E, Schnell T: Body integrity identity disorder: development and evaluation of an inventory for the assessment of the severity. Am J Appl Psychol 2015;4:76-82.

28 Frick C, Lang S, Kotchoubey B, Sieswerda S, Dinu-Biringer R, Berger M, Barnow S: Hypersensitivity in borderline personality disorder during mindreading. PLoS One 2012;7:e41650.

29 Tokaj A: Mit männlichen Augen. 2012. http://www.spektrum.de/news/mit-maennlichenaugen/1161229 (accessed June 6, 2016).

-30 Fioretti AB, Varakliotis T, Cantagallo M, Eibenstein A: Severe hyperacusis, photophobia, and skin hypersensitivity. Case Rep Otolaryngol 2016;2016:2570107.

31 McTeague LM, Lang PJ, Wangelin BC, Laplante MC, Bradley MM: Defensive mobilization in specific phobia: fear specificity, negative affectivity, and diagnostic prominence. Biol Psychiatry 2012;72:8-18. Bartoshuk LM: Comparing sensory experiences across individuals: recent psychophysical advances illuminate genetic variation in taste perception. Chem Senses 2000;25:447-460.

33 Gomes E, Pedroso FS, Wagner MB: Auditory hypersensitivity in the autistic spectrum disorder. Pro Fono 2008;20:279-284.

-34 Nordin S, Neely G, Olsson D, Sandström M: Odor and noise intolerance in persons with self-reported electromagnetic hypersensitivity. Int J Environ Res Public Health 2014;11:8794-8805.

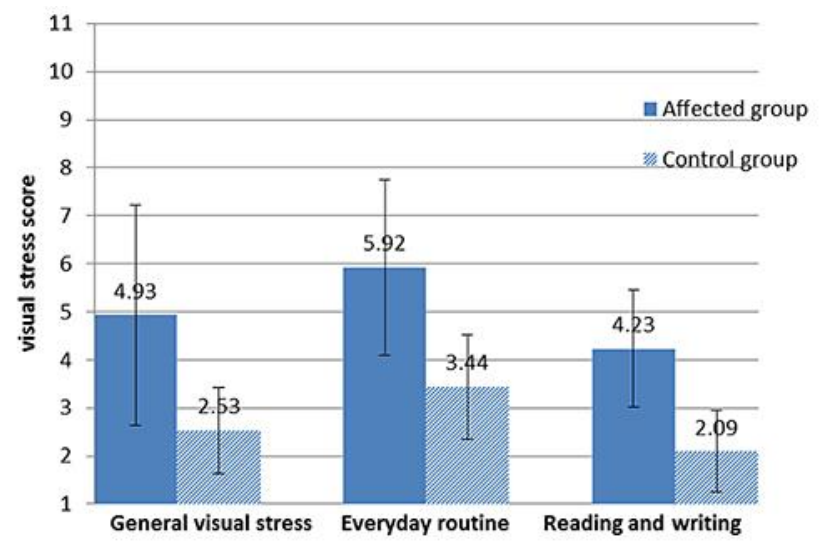

Fig. 1. Visual stress (categories 1-3) of affected people ( $n=5$, plain) versus the control group $(n=35$, striped) on a scale from 1 = no visual stress to 11 = max. visual stress (mean and standard deviation). 


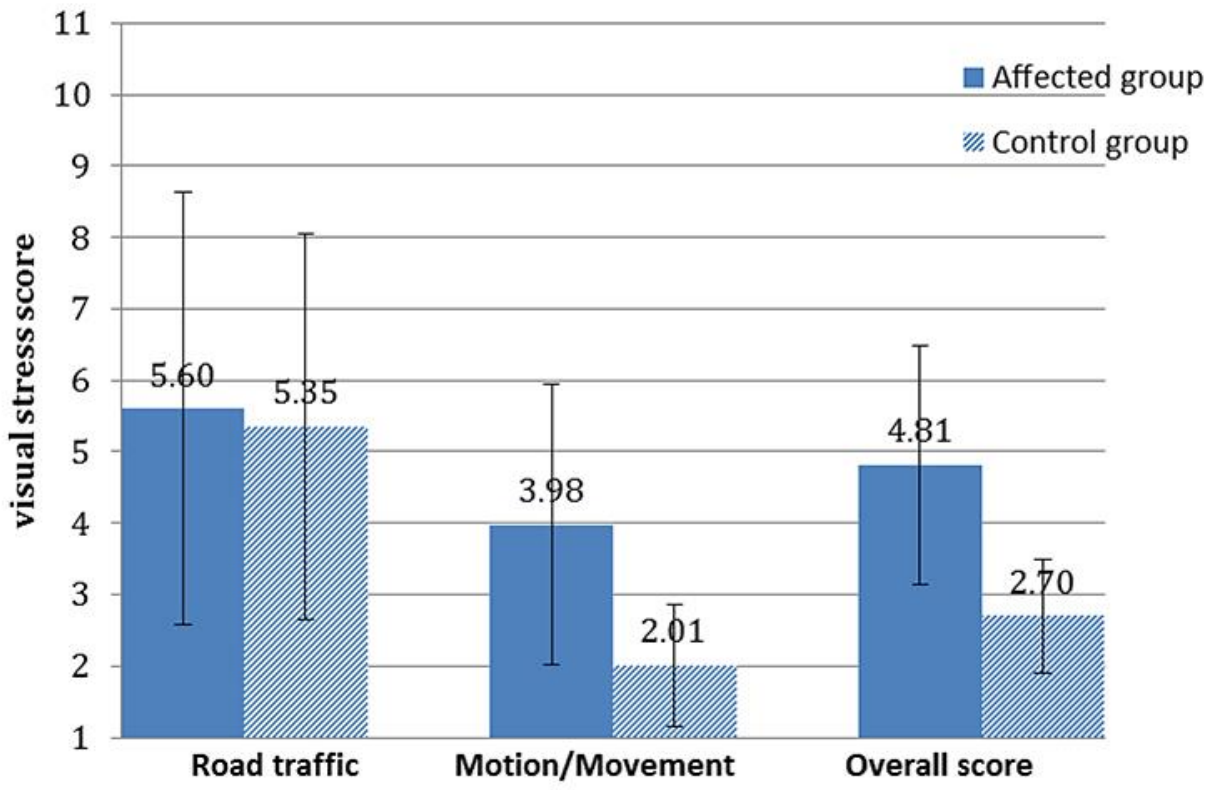

Fig. 2. Visual stress (categories 4-5 and overall score) of affected people ( $n=5$, plain) versus the control group ( $n=35$, striped) on a scale from $1=$ no visual stress to $11=$ max. visual stress (mean and standard deviation). 


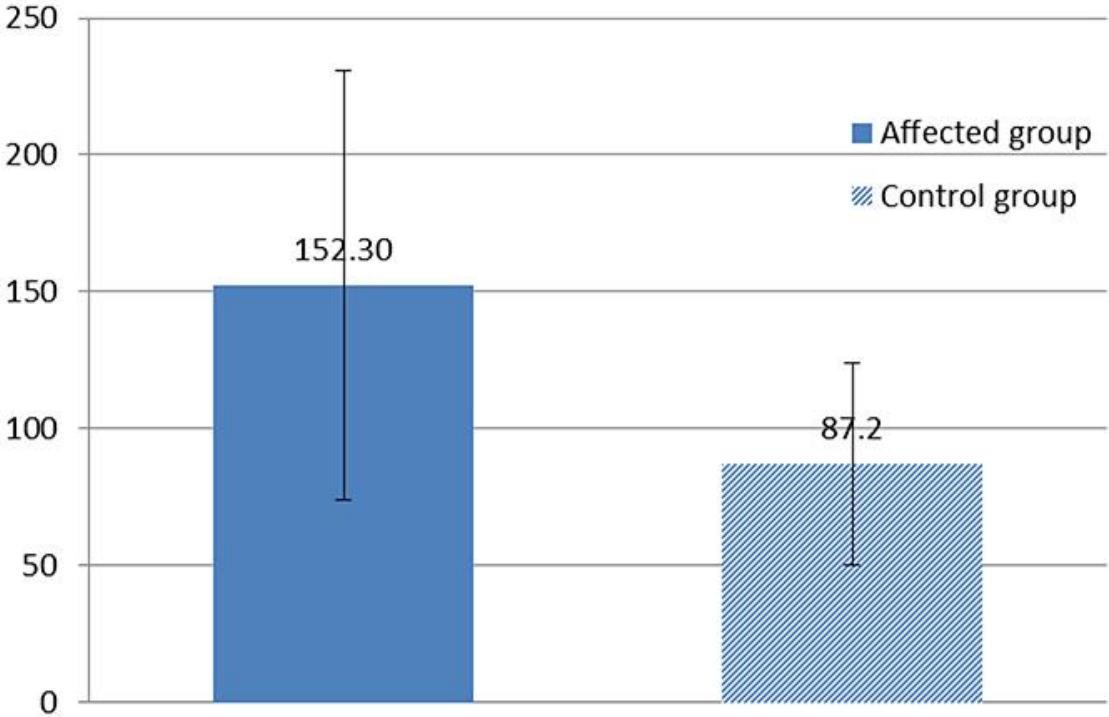

Fig. 3. Time period (s) needed to solve 4 hidden object game trials in the affected group ( $n=5$, plain) and in the controls ( $n=28$, striped). Mean and standard deviation are shown.

Table 1. Visual stress of affected people versus control group: average, standard deviation, and results of Mann-Whitney test

\begin{tabular}{llll}
\hline Category & $\begin{array}{l}\text { Affected group } \\
(n=5)\end{array}$ & $\begin{array}{l}\text { Control group } \\
(n=35)\end{array}$ & $\begin{array}{l}\text { Significance } \\
\text { (one-sided) }\end{array}$ \\
\hline General visual stress (11 items) & $4.93 \pm 2.29$ & $2.53 \pm 0.90$ & $p=0.011^{*}$ \\
Everyday routine (20 items) & $5.92 \pm 1.82$ & $3.44 \pm 1.08$ & $p=0.002^{* *}$ \\
Reading and writing (21 items) & $4.23 \pm 1.22$ & $2.09 \pm 0.85$ & $p=0.002^{* *}$ \\
Road traffic (5 items) & $5.60 \pm 3.03$ & $5.35 \pm 2.70$ & $p=0.196 \mathrm{~ns}$ \\
Motion/movement (18 items) & $3.98 \pm 1.97$ & $2.01 \pm 0.85$ & $p=0.009^{* *}$ \\
\hline Overall score (75 items) & $4.81 \pm 1.67$ & $2.70 \pm 0.80$ & $p=0.004^{* *}$ \\
\hline
\end{tabular}




\section{Case Reports in Ophthalmology}

\begin{tabular}{l|l} 
Case Rep Ophthalmol 2017;8:124-136 \\
\hline DOI: $10.1159 / 000456709$ & $\begin{array}{l}\text { (c) 2017 The Author(s). Published by S. Karger AG, Basel } \\
\text { www.karger.com/cop }\end{array}$ \\
\hline
\end{tabular}

Gutschke et al.: An Overwhelming Desire to Be Blind: Similarities and Differences between Body Integrity Identity Disorder and the Wish for Blindness

Table 2. Intensity of the desire for blindness and intensity of visual stress

\begin{tabular}{llll}
\hline Participant & $\begin{array}{l}\text { Intensity of the } \\
\text { desire for blindness }\end{array}$ & $\begin{array}{l}\text { Classification according } \\
\text { to Fischer [27] }\end{array}$ & $\begin{array}{l}\text { Intensity of } \\
\text { visual stress }\end{array}$ \\
\hline A & 5.00 & medium & 5.75 \\
B & 4.86 & medium & 4.61 \\
C & 2.71 & weak & 3.95 \\
D & 6.14 & strong & 2.71 \\
E & 5.07 & medium & 7.05 \\
\hline
\end{tabular}

